\title{
Effect of N Atoms on O-Atom Reactivity with Carbon
}

\author{
Chenbiao Xu and Timothy K. Minton* \\ Department of Chemistry and Biochemistry \\ Montana State University \\ Bozeman, MT 59717 United States
}

\section{Introduction}

The dominant reactive species in the shock layer of a hypersonic vehicle that flies through air is atomic oxygen. Thus, a great deal of effort has been spent to understand the oxidation of carbon in our laboratory.[1-3] The data from this effort has been used in the formulation of oxygen-carbon ablation models.[4-6] However, the development of an air-carbon ablation model requires an understanding of the effects of $\mathrm{N}$ atoms and their reactions on the reactivity of $\mathrm{O}$ atoms with carbon. Even if the N-atom reactivity with carbon is relatively small (as suggested by our molecular beam studies of $\mathrm{N}$ atoms on vitreous carbon[1,7]) or if the $\mathrm{N}$-atom flux onto the surface is low, it is possible that the presence of $\mathrm{N}$ atoms might block reactive sites or act as a catalyst to promote O-carbon reactions. Without data, we can only speculate about the effect of $\mathrm{N}$ atoms on the reactivity of $\mathrm{O}$ atoms with carbon. Therefore, we have undertaken a series of experiments to determine how bombardment of a carbon surface with both $\mathrm{N}$ and $\mathrm{O}$ atoms enhances or inhibits the reaction of $\mathrm{O}$ atoms with a carbon surface to produce $\mathrm{CO}$, which is the most important oxidation reaction on a high-temperature carbon surface.

*Corresponding author. Present address: Smead Department of Aerospace Engineering Sciences, University of Colorado, Boulder, Colorado 80303 United States; E-mail: tminton@colorado.edu; ORCID: 0000-0003-4577-7879 


\section{Experimental Approach}

The experiments used a beam-surface scattering method with a pulsed $(2 \mathrm{~Hz})$ hyperthermal molecular beam that was produced by laser detonation. Figure 1 shows a diagram of the experimental set-up, which is very similar to that used for earlier studies of $\mathrm{O}$ - and $\mathrm{N}$-atom reactions with vitreous carbon.[1,7]

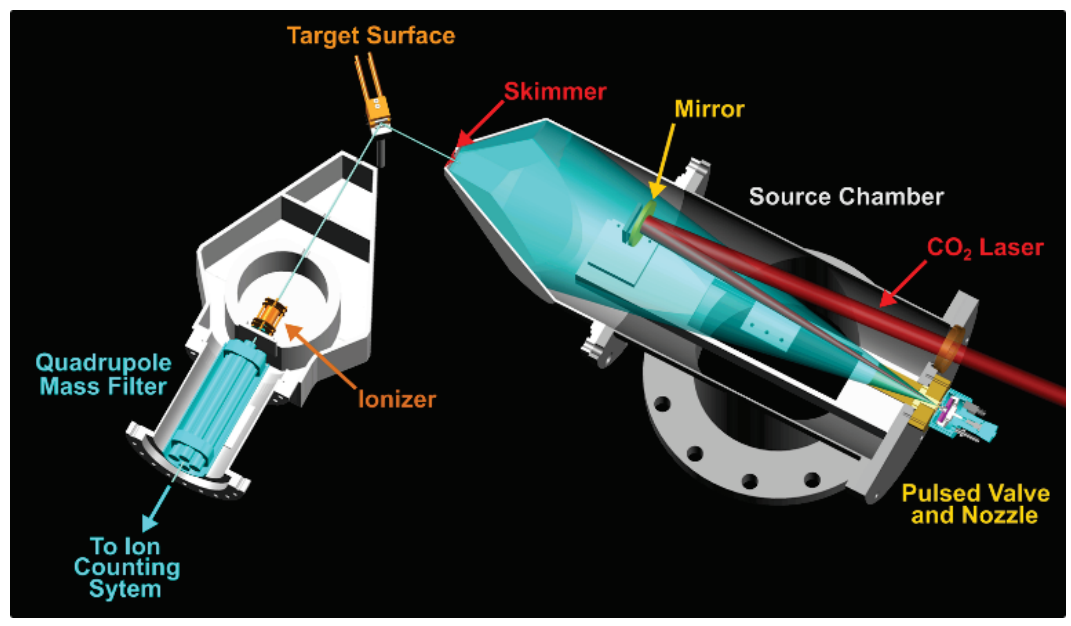

Figure 1. Schematic diagram of the set-up for the molecular beamsurface scattering experiment, with the hyperthermal beam source, the heated sample, and rotatable mass spectrometer detector.

The laser-detonation source was used to produce a beam containing the five species in nonequilibrium air: $\mathrm{N}, \mathrm{N}_{2}, \mathrm{O}, \mathrm{O}_{2}$, and $\mathrm{NO}$. Although $\mathrm{O}$ and $\mathrm{N}$ are expected to be the only relevant reactive species in such a mixture, all of these species would be present in the boundary layer of a hypersonic vehicle, so it was reasonable to use a beam that contained these species when collecting data that could be used for an air-carbon ablation model. $\mathrm{N}_{2}$ is not expected to react with carbon. $\mathrm{O}_{2}$ will react with carbon slowly, but its reactivity is two or more orders of magnitude lower than that of $\mathrm{O}$ and probably at least an order of magnitude lower than that of N.[3] The reactivity of NO is unknown, and being a radical, one might expect it to react at least slowly, but we assume that it reacts much more slowly than $\mathrm{O}$ and $\mathrm{N}$. The laser detonation hyperthermal molecular beam source was used to produce a beam containing all five species with the use of a precursor gas that contained roughly $40 \% \mathrm{O}_{2}$ and $60 \% \mathrm{~N}_{2}$. The mass spectrometer was used to interrogate the beam, 
and the translational energy distributions and mole fractions of each species in the beam were determined (see Figure 2). As may be seen in Figure 2, the total mole fraction of $\mathrm{O}$ atoms in all forms is 0.36 and the total mole fraction of $\mathrm{N}$ atoms in all forms is 0.64 . These fractions differ somewhat from what is expected based on the mole fractions of $\mathrm{O}_{2}$ and $\mathrm{N}_{2}$ in the precursor gas mixture. It is possible that the nature of the gas dynamics as the beam is formed favors nitrogen species slightly over oxygen species along the molecular beam axis where the gas was sampled. Also, uncertainties in the mass-dependent detection sensitivities, which we attempted to correct for, may contribute to deviations between the measured and expected $\mathrm{O}$ and $\mathrm{N}$ mole fractions. The average velocity of the species in the beam was $6600 \mathrm{~m} \mathrm{~s}^{-1}$. In addition to the mixed oxygen/nitrogen beam, additional pulsed hyperthermal beams were prepared by the same method but with either pure $(100 \%) \mathrm{O}_{2}$ or pure $(100 \%) \mathrm{N}_{2}$ as the precursor gas. The resulting hyperthermal beams after laser detonation contained mixtures of approximately $85 \% \mathrm{O}$ and $15 \% \mathrm{O}_{2}$ or $36 \% \mathrm{~N}$ and $64 \% \mathrm{~N}_{2}$, respectively, and they had the same average velocity as the mixed nitrogen/oxygen beam. The beams were directed at a vitreous carbon surface, and time-of-flight (TOF)

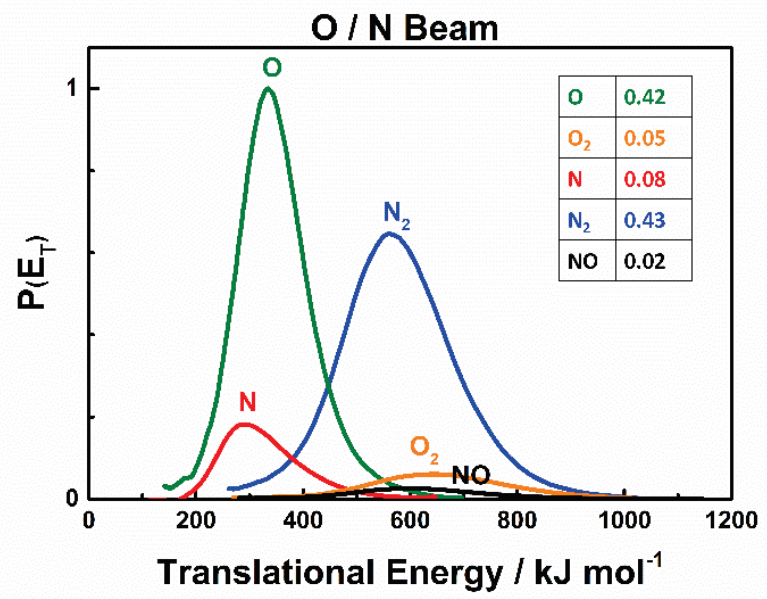

Figure 2. Translational energy distributions and mole fractions of atomic and molecular species in mixed oxygen/nitrogen hyperthermal molecular beam.

distributions were collected with a rotatable mass spectrometer detector, using the experimental set-up shown in Figure 1, which is identical to the set-up described in Ref. 7. The flux of reactive atoms onto the surface is estimated to be on the order of $10^{15}$ atoms $\mathrm{cm}^{-2} \mathrm{~s}^{-1}$, regardless of the 
beam used. Beam-surface scattering experiments were conducted with three focus sample temperatures, $T_{s}=1100 \mathrm{~K}, 1400 \mathrm{~K}$, and $1700 \mathrm{~K}$. TOF distributions were collected with an angle of incidence of $\theta_{i}=60^{\circ}$ and a final (or detection) angle of $\theta_{f}=45^{\circ}$. The TOF distributions may exhibit two components,[8,9] (1) impulsive scattering (IS), where the incident atoms or molecules or their reaction products scatter on a time scale too short for thermal equilibrium to be achieved, and (2) thermal desorption (TD), where the incident atoms or molecules or their reaction products desorb in thermal equilibrium with the surface after losing all "memory" of the incident angle and velocity of the impinging species. IS products tend to scatter with hyperthermal translational energies in a lobular angular distribution that is peaked far from the surface normal. TD products generally scatter with a Maxwell-Boltzmann distribution of velocities, with maximum flux toward the surface normal. As has been shown previously, when hyperthermal $\mathrm{O}$ and $\mathrm{O}_{2}$ strike a vitreous carbon surface, they may scatter non-reactively through both IS and TD pathways.[1] On the other hand, reactively scattered $\mathrm{CO}$ and $\mathrm{CO}_{2}$ desorb mostly thermally even when the incident O-atom energy is hyperthermal.[1] When $\mathrm{N}$ and $\mathrm{N}_{2}$ that are incident on a vitreous carbon surface at hyperthermal energies, they scatter mainly non-thermally through IS pathways (perhaps with a minor fraction of TD),[7] whereas the reactive $\mathrm{CN}$ product desorbs solely in thermal equilibrium even when the incident energy is hyperthermal.[7]

\section{Results and Analysis}

For each of the three focus sample temperatures, the protocol for data collection was the same, and results for $T_{s}=1700 \mathrm{~K}$ are used here as an example. The vitreous carbon sample was first annealed in vacuum for 40 minutes at $1900 \mathrm{~K}$. Through much experience, we have determined that this annealing condition removes all oxygen and nitrogen that might be adsorbed and leaves a 
pure carbon surface. Then, the sample temperature was reduced to the particular focus temperature. The mixed oxygen/nitrogen beam was then directed at the surface, and TOF distributions were collected at $m / z=16\left(\mathrm{O}^{+}\right), 28\left(\mathrm{CO}^{+}, \mathrm{N}_{2}{ }^{+}\right)$, and $32\left(\mathrm{O}_{2}{ }^{+}\right)$. Note that the signal at $m / z=28$ contains non-reactively scattered $\mathrm{N}_{2}$, as well as reactively scattered $\mathrm{CO}$ from the reaction of $\mathrm{O}$ with carbon to produce $\mathrm{CO}$. After data collection with the mixed oxygen/nitrogen beam, the pure nitrogen $\left(\mathrm{N} / \mathrm{N}_{2}\right)$ beam was directed at the surface, and a TOF distribution was collected at $\mathrm{m} / \mathrm{z}$ $=28\left(\mathrm{~N}_{2}{ }^{+}\right.$, black curve in Figure 3$)$. The main peak at $m / z=28$ can only come from impulsively scattered $\mathrm{N}_{2}$,[7] so this peak was scaled to match the peak of the $m / z=28$ signal from the TOF distribution collected with the mixed oxygen/nitrogen beam, whose main peak at $\mathrm{m} / \mathrm{z}=28$ must also come only from impulsive scattering of $\mathrm{N}_{2}$ (blue and red curves of Figure 3). Because the

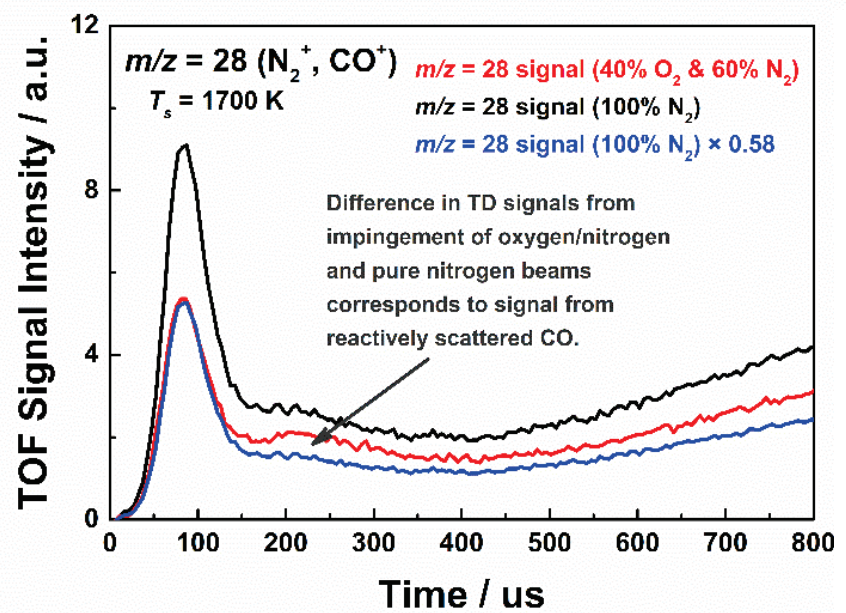

Figure 3. Time-of-flight distributions collected at $\mathrm{m} / \mathrm{z}$ $=28$ for the oxygen/nitrogen beam (red) and the pure nitrogen beam (black). The blue curve shows the distribution for the pure nitrogen beam that has been scaled by a factor of 0.58 to match the magnitude of the peak in the distribution obtained with the oxygen/nitrogen beam. The difference between the red and blue curves should be the time-of-flight distribution of reactively scattered $\mathrm{CO}$ when the oxygen/nitrogen beam bombards the surface. Note that " $100 \% \mathrm{~N}_{2}$ " refers to the beam prepared with pure $\mathrm{N}_{2}$ as the precursor gas, resulting in a beam containing $\mathrm{N}$ and $\mathrm{N}_{2}$ with a mole ratio of $0.36 / 0.64$, respectively.

$m / z=28$ TOF distribution collected for the mixed oxygen/nitrogen beam contains components corresponding to $\mathrm{N}_{2}$ and $\mathrm{CO}$, the difference between the red and blue curves in Figure 3 should be the TOF distribution for $\mathrm{CO}$, which is expected to result from TD (i.e., slower) products. The long tails in the TOF distributions (at times $>400 \mu \mathrm{s}$ ) come from $\mathrm{N}_{2}$ molecules that effuse from the source chamber after the hyperthermal pulse. These slowly effusing molecules can scatter from the sample in a TD process and lead to substantial signal in the detector. The fact that the long 
tails in the blue and red curves at long times do not match suggests that the effusion of $\mathrm{N}_{2}$ from the source chamber has slightly different characteristics when $\mathrm{N}_{2}$ is in the mixed oxygen/nitrogen beam than when it is in the pure nitrogen $\left(\mathrm{N} / \mathrm{N}_{2}\right)$ beam. Thus, the subtraction of the blue curve from the red curve in Figure 3 will yield signal at long times that is an artifact of the different characteristics of the effusive background. Nevertheless, the TD CO signal detected at $m / z=28$ is expected to have a peak between 200 and $300 \mu$ s in the TOF distribution, so this signal should only be minimally affected by the effusive background when the blue curve is subtracted from the red curve. Following data collection with the nitrogen $\left(\mathrm{N} / \mathrm{N}_{2}\right)$ beam, the sample was once again annealed at $1900 \mathrm{~K}$, without exposure from any beam. Then the sample temperature was reduced to the particular focus temperature and the oxygen $\left(\mathrm{O} / \mathrm{O}_{2}\right)$ beam prepared from pure $\mathrm{O}_{2}$ precursor gas was directed at the surface, and TOF distributions were collected at $m / z=16\left(\mathrm{O}^{+}\right), 28\left(\mathrm{CO}^{+}\right)$, and $32\left(\mathrm{O}_{2}{ }^{+}\right)$. The $m / z=32$ distribution was used to correct the $m / z=16$ distribution for signal

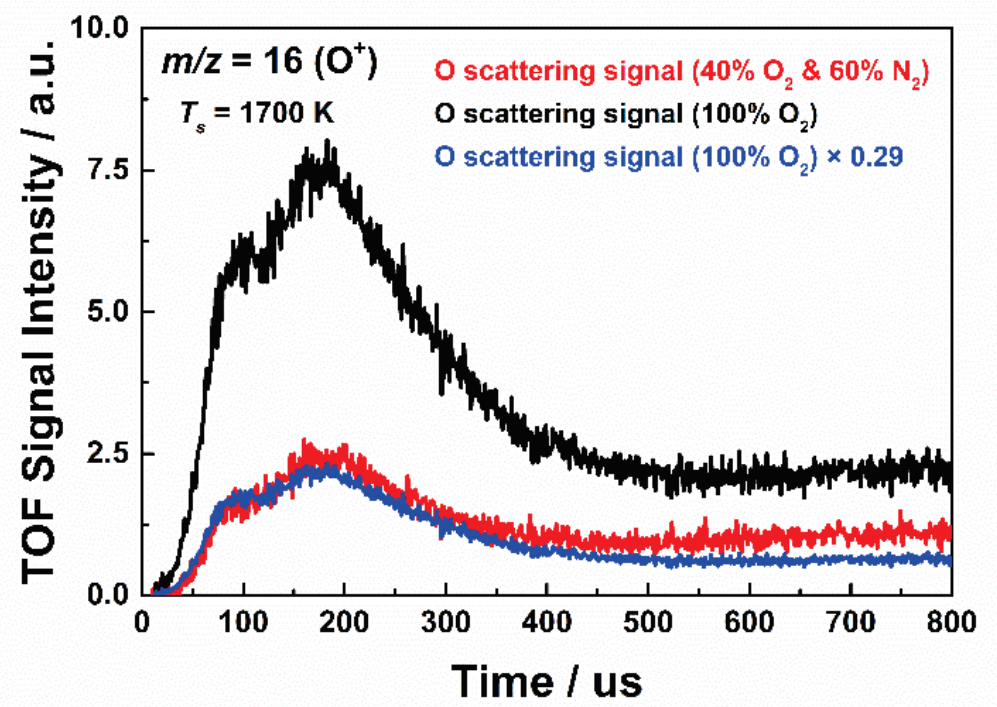

Figure 4. Time-of-flight distributions collected at $m / z=16$ for the pure oxygen beam (black) and the oxygen/nitrogen beam (red). The blue curve shows the distribution for the pure oxygen beam that has been scaled by a factor of 0.29 to match the magnitude of the distribution obtained with the mixed oxygen/nitrogen beam. This scaling factor is applied to the CO signal (detected at $\mathrm{m} / \mathrm{z}=28$ ) from the pure oxygen beam to predict the time-offlight distribution for $\mathrm{CO}$ from the oxygen/nitrogen beam, in the absence of any effect of nitrogen. Note that " $100 \%$ $\mathrm{O}_{2}$ " refers to the beam prepared with pure $\mathrm{O}_{2}$ as the precursor gas, resulting in a beam containing $\mathrm{O}$ and $\mathrm{O}_{2}$ with a mole ratio of $0.85 / 0.15$, respectively. 
arising from dissociative ionization of $\mathrm{O}_{2}$ in the electron-bombardment ionizer of the detector $\left(\mathrm{O}_{2}\right.$ $\left.+e^{-} \rightarrow \mathrm{O}^{+}+\mathrm{O}+2 e^{-}\right)$such that the $m / z=16$ TOF distribution only represented $\mathrm{O}$ atoms that exited the surface without net reaction. The $m / z=28$ distribution could only result from the reaction of $\mathrm{O}$ with carbon to produce $\mathrm{CO}$. In order to predict the magnitude of the $\mathrm{CO}$ signal that should come from the oxygen/nitrogen beam in the absence of any effect of nitrogen, it was necessary to determine the relative flux of $\mathrm{O}$ in the mixed oxygen/nitrogen beam compared to that in the pure oxygen beam. This was determined by scaling the $m / z=16$ signal from the pure oxygen beam to match the $m / z=16$ signal from the mixed oxygen/nitrogen beam (Figure 4). TOF distributions derived from the pure oxygen beam (scaled appropriately) and the mixed oxygen/nitrogen beam are shown in Figure 5. When the $\mathrm{CO}$ signal derived from the O-atom flux in the mixed oxygen/nitrogen beam is multiplied by a factor of 1.6, it matches the actual $\mathrm{CO}$ signal that was measured for the oxygen/nitrogen beam. This result suggests that the presence of nitrogen on the surface enhances the O-atom reactivity with carbon to produce $\mathrm{CO}$ by a factor of 1.6. In fact, this factor was the same for experiments with focus sample temperatures of $T_{s}=1400 \mathrm{~K}$ and $1100 \mathrm{~K}$. In addition, an enhancement factor of about 1.4 has been found in an additional experiment with a precursor gas mixture of $60 \% \mathrm{O}_{2}$ and $40 \% \mathrm{~N}_{2}$ and a surface temperature of $1400 \mathrm{~K}$. This mixture produced a hyperthermal beam with mole fractions of $0.52(\mathrm{O}), 0.05\left(\mathrm{O}_{2}\right), 0.02(\mathrm{~N}), 0.39\left(\mathrm{~N}_{2}\right)$, and $0.02(\mathrm{NO})$. The fact that a decrease in the mole fraction of $\mathrm{N}$ in the beam from 0.08 to 0.02 only decreased the enhancement factor from 1.6 to 1.4 suggests that the observed enhancement in the $\mathrm{O}$-atom reactivity to produce $\mathrm{CO}$ is achieved with only a tiny fraction of $\mathrm{N}$ atoms compared to $\mathrm{O}$ atoms and that the enhancement may not be very sensitive to the fraction of $\mathrm{N}$ atoms. 


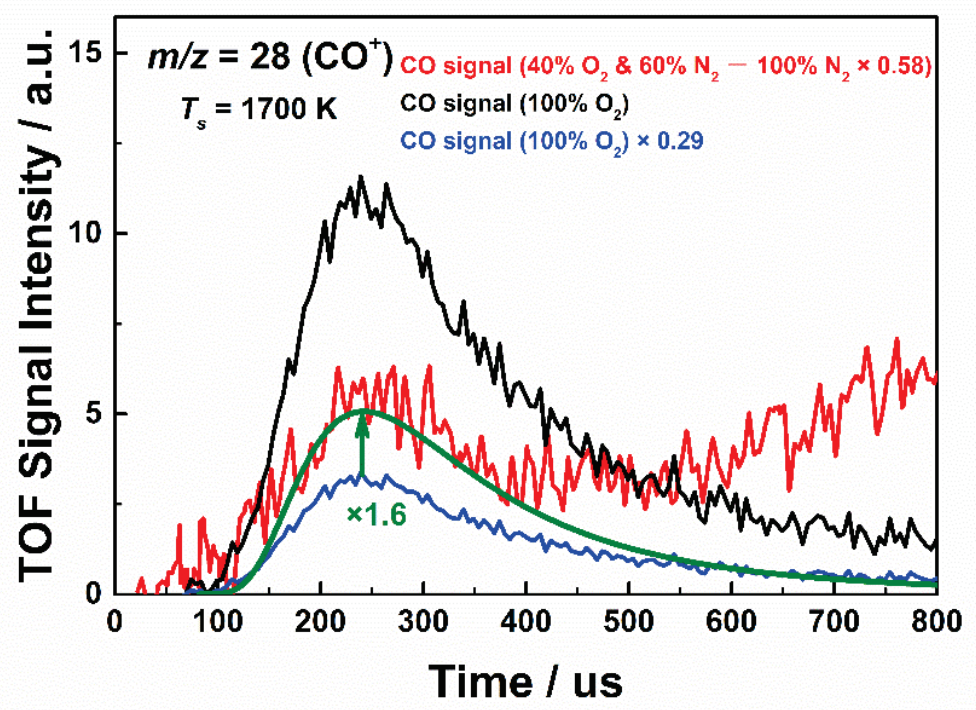

Figure 5. Time-of-flight distributions collected or derived for $\mathrm{CO}$ (detected at $\mathrm{m} / \mathrm{z}=28$ ). The $\mathrm{CO}$ signal for the pure oxygen $\left(\mathrm{O} / \mathrm{O}_{2}\right)$ beam (black) has been scaled by a factor of 0.29 to give the predicted CO TOF distribution from the mixed oxygen/nitrogen beam (blue) in the absence of any effect of nitrogen. The red curve is the actual $\mathrm{CO}$ signal detected with the mixed oxygen/nitrogen beam. The long tail in the red distribution is the result of a slight difference in the shapes of the time-of-flight distributions collected at $m / z=28$ with the oxygen/nitrogen beam and the pure nitrogen $\left(\mathrm{N} / \mathrm{N}_{2}\right)$ beam (see Figure 3), which presumably results from slightly different characteristics in the long-time effusion of $\mathrm{N}_{2}$ from the source chamber for the two beams. When the $\mathrm{CO}$ signal derived under the assumption of no effect from nitrogen is multiplied by a factor of 1.6, the resulting signal magnitude matches that of the actual CO signal measured with the mixed oxygen/nitrogen beam (green curve).

\section{Conclusion}

Molecular beam-surface scattering experiments have been used to study the effect of $\mathrm{N}$ atoms on the reactivity of $\mathrm{O}$ atoms with a high-temperature carbon surface to produce $\mathrm{CO}$. The $\mathrm{CO}$ flux produced from bombardment of a vitreous carbon surface by a beam containing $\mathrm{N}$ and $\mathrm{O}$ atoms was compared with the $\mathrm{CO}$ flux produced from bombardment by beams containing either $\mathrm{N}$ or $\mathrm{O}$ atoms. The presence of small mole fractions of $\mathrm{N}$ atoms of $0.02-0.08$ enhanced the reactivity of $\mathrm{O}$ atoms by a factor of 1.4-1.6 in the range of surface temperatures from 1100 to 1700 K. A detailed explanation of the observed results requires more study, but it appears that $\mathrm{N}$ atoms can act as a catalyst to increase the reactivity of $\mathrm{O}$ with carbon and that a relatively low flux of $\mathrm{N}$ atoms may be sufficient to saturate the catalytic effect. This catalytic effect seems to be fairly 
insensitive to the surface temperature, at least over the temperature range used in this study. This observation is important in the development of finite-rate models of air-carbon ablation during hypersonic flight.

\section{Acknowledgements}

This work was supported by the U.S. Air Force office of Scientific Research: FA 9550-170057. The authors are grateful to Prof. Thomas Schwartzentruber for many helpful discussions.

\section{References}

[1] Murray, V. J.; Marshall, B. C.; Woodburn, P. J.; Minton, T. K. Inelastic and Reactive Scattering Dynamics of Hyperthermal $\mathrm{O}$ and $\mathrm{O}_{2}$ on Hot Vitreous Carbon Surfaces. J. Phys. Chem. C 2015, $119,14780-14796$.

[2] Murray, V. J.; Smoll, E. J.; Minton, T. K. Dynamics of Graphite Oxidation at High Temperature. J. Phys. Chem. C 2018, 122, 6602-6617.

[3] Murray, J. J.; Recio, P.; Caracciolo, A.; Miossec, C.; Balucani, N.; Casavecchia, P.; Minton, T. K. Oxidation and Nitridation of Vitreous Carbon at High Temperatures. Carbon 2020. DOI: 10.1016/j.carbon.2020.05.076.

[4] S. Poovathingal, T.E. Schwartzentruber, V.J. Murray, T.K. Minton, G.V. Candler, Finite-rate oxidation model for carbon surfaces from molecular beam experiments, AIAA J. 2017, 55, 16441658.

[5] K. Swaminathan-Gopalan, A. Borner, V.J. Murray, S. Poovathingal, T.K. Minton, N.N. Mansour, K.A. Stephani, Development and validation of a finite-rate model for carbon oxidation by atomic oxygen, Carbon 2018, 137, 313-332.

[6] K. Swaminathan-Gopalan, K.A. Stephani, Construction of finite rate surface chemistry models from pulsed hyperthermal beam experimental data, AIP Adv., 2019, 9, 035246.

[7] Murray, V. J.; Minton, T. K. Gas-Surface Interactions of Atomic Nitrogen on Carbon. Carbon 2019, 150, 85-92.

[8] Hurst, J. E.; Becker, C. A.; Cowin, J. P.; Janda, K. C.; Wharton, L.; Auerbach, D. J. Observation of Direct Inelastic Scattering in the Presence of Trapping-Desorption Scattering: Xe on Pt(111). Phys. Rev. Lett. 1979, 43, 1175

[9] Rettner, C. T.; Schweizer, E. K.; Mullins, C. B. Desorption and Trapping of Argon at a 2HW(100) Surface and a Test of the Applicability of Detailed Balance to a Nonequilibrium System. J. Chem. Phys. 1989, 90, 3800. 